\title{
Automatic morphometry of nerve histological sections
}

\author{
E. Romero ${ }^{\mathrm{a}}$, O. Cuisenaire ${ }^{\mathrm{b}}$, J.F. Denef ${ }^{\mathrm{c}}$, J. Delbeke ${ }^{\mathrm{a}}$, B. Macq ${ }^{\mathrm{b}}$, C. Veraart ${ }^{\mathrm{a}, *}$ \\ a Neural Rehabilitation Engineering Laboratory, Université catholique de Louvain, 54 Avenue Hippocrate UCL-54.46, B-1200 Brussels, Belgium \\ ${ }^{\mathrm{b}}$ Communication and Remote Sensing Laboratory, Université catholique de Louvain, 2, place du Levant, B-1348 Louvain-la-Neuve, Belgium \\ ${ }^{\mathrm{c}}$ Histology Laboratory, Université catholique de Louvain, 52 Avenue Mounier UCL-52.29, B-1200 Brussels, Belgium
}

Received 11 October 1999; received in revised form 14 January 2000; accepted 15 January 2000

\begin{abstract}
A method for the automatic segmentation, recognition and measurement of neuronal myelinated fibers in nerve histological sections is presented. In this method, the fiber parameters i.e. perimeter, area, position of the fiber and myelin sheath thickness are automatically computed. Obliquity of the sections may be taken into account. First, the image is thresholded to provide a coarse classification between myelin and non-myelin pixels. Next, the resulting binary image is further simplified using connected morphological operators. By applying semantic rules to the zonal graph axon candidates are identified. Those are either isolated or still connected. Then, separation of connected fibers is performed by evaluating myelin sheath thickness around each candidate area with an Euclidean distance transformation. Finally, properties of each detected fiber are computed and false positives are removed. The accuracy of the method is assessed by evaluating missed detection, false positive ratio and comparing the results to the manual procedure with sampling. In the evaluated nerve surface, a $0.9 \%$ of false positives was found, along with $6.36 \%$ of missed detections. The resulting histograms show strong correlation with those obtained by manual measure. The noise introduced by this method is significantly lower than the intrinsic sampling variability. This automatic method constitutes an original tool for morphometrical analysis. (c) 2000 Elsevier Science B.V. All rights reserved.
\end{abstract}

Keywords: Morphometry; Nerve histology; Axon parameters; Axon recognition; Mathematical morphology; Connected operators

\section{Introduction}

Morphometric studies on nerve trunks are valuable tools in detecting development or pathological abnormalities (Gutmann and Sanders, 1942; Cragg and Thomas, 1961; Ohnishi et al., 1977; Dyck et al., 1984; Jacobs and Love, 1985). They have also been broadly used in experimental nerve research (Pick, 1956; Buchnal and Behse, 1978). Usually, these studies are focused on myelinated fiber size and number, although fiber perimeter and myelin thickness sheaths are also considered as useful parameters. Many investigators (Duncan, 1934; Fernand and Young, 1951; Wendell-Smith and Williams, 1959; Donovan, 1967) have used manual methods, for instance at measuring two different fiber diameters and taking their mean as a fiber size estimator. Clearly, the amount of work demanded by this

\footnotetext{
* Corresponding author. Tel.: +32-2-7645446; fax: +32-2 7649422 .

E-mail address: veraart@gren.ucl.ac.be (C. Veraart)
}

kind of approach was greatly dependent on the nerve cross surface and fiber size population. In consequence, in large experimental studies too few nerve profiles can be measured. Also, there is an unavoidable bias introduced by the profile selection the experimenter made from the entire population.

Attempts to increase the number of measurements led investigators to employ semi-automatic systems (Dunn et al., 1975) resulting in significant computation time reduction, but still unsuitable for study nerves with a large fiber population i.e. optic nerves (Kupfer et al., 1967; Treff et al., 1972). Even though adequate sampling schemes have permitted to attack this problem at a reasonable effort-time cost, much of the measurements keep to be performed by the experimenter. Many different sampling schemes are described in the literature (Mathieu et al., 1981; Müller et al., 1981; Mayhew and Sharma, 1984a,b; Torch et al., 1989) for estimating parameters as fiber diameter, fiber surface or perimeter, in either homogeneous or heterogeneous fiber distributions. The amount of work needed to achieve these 
estimations is variable according to different investigators. Some authors (Mayhew and Sharma, 1984a) find a good estimation of fiber diameter with only 185 fibers among some 2900 as in the rat tibial nerve, whereas others claim that a minimum of $50 \%$ of the entire fiber population (Torch et al., 1989) is needed for fiber area and perimeter estimations. In the former case (Mayhew and Sharma, 1984a), a fair estimate of the fiber diameter distribution takes $16 \mathrm{~min}$. In the latter case (Torch et al., 1989), e.g. the optic nerve it may mean half a million measurements.

Lately, stereological techniques as the fractionator (Gundersen, 1986) have been successfully used for calculating the number of myelinated fibers (Mayhew, 1988), reducing considerably the task. These sampling procedures are based on unbiased estimation of a particular parameter, whereby all nerve fibers have the same probability of being examined. Larsen (1998) has extended its application to other nerve parameters such as the area, the perimeter and myelin thickness. Although, it deals well with a precise estimation of these parameters and is far less time consuming than complete tracing of fibers, it remains still an important work for an operator in very inhomogeneous nerves, where larger number of samples ('counting frames') are needed, or in studies where many nerves have to be used, as it is frequent the case in a experiment design conceived to unmask a particular condition.

As a consequence, an automatic image analysis system deserves to be considered. Combination with any adequate sampling scheme should optimize its efficiency and reduce the experimenter work. Automatic approaches have been used in several histological applications. Morphological recognition algorithms have been introduced to isolate regions of interest in certain images (Garbay, 1986). Such segmentation algorithms usually proceed in two steps. First, the image is analyzed with a local operator, which classifies pixels according to the various tissue characteristics expected to be found. Next, the image is analyzed at the structural level using a variety of tools such as region growing segmentation (Jain et al., 1980), grouping of edge elements (Garbay, 1986), or mathematical morphology (Thiran and Macq, 1996). Unfortunately, none of these methods can handle multi-part objects such as axons surrounded by a myelin sheath. Lately, several authors have relied on active contour models, or snakes (Amini et al., 1990; Fok et al., 1996; Elmoataz et al., 1998), to handle both local and structural analysis in one step. After detecting candidates through a global tool such as the Hough transform, each region of interest is processed individually with an explicit active contour model evolving towards the real contours of the structure. Unfortunately, such method may be too computationally expensive. Furthermore, it is unclear whether any of these models could handle the large size variability encountered in some fibers.

The present study proposes and evaluates an original automatic method for segmentation, recognition, and measurement of nerve fibers based on connected morphological operators to identify candidate areas, and on the Euclidean distance transformation to separate fiber aggregates. This procedure results in a reliable estimation of the total number of myelinated axons, the distribution of the myelin sheath thickness and area, as well as the fiber diameter, area and perimeter. The method also reconstructs the position of each measured fiber in nerve co-ordinates and corrects for section obliquity.

\section{Methods}

\subsection{Animal and tissue preparation}

The sciatic nerve used in this study was obtained from a female cat $(3250 \mathrm{~g})$ used as control in an unrelated experiment. The cat was anaesthetised with sodium pentobarbital (30 mg/kg I.M.). A $14 \mathrm{G}$ catheter was inserted in the abdominal aorta, between the renal branches and iliac bifurcation. A solution of 10000 units of heparin and dextran $3.5 \%$ in a PBS phosphate buffer was given for $1 \mathrm{~min}$ as a prewash at a constant flow rate of $250 \mathrm{ml} / \mathrm{min}$, followed by 21 of phosphatebuffered para-formaldehyde solution. The sciatic nerve was then dissected and a short sample was excised at about $4 \mathrm{~cm}$ proximal to the bifurcation into the tibial and peroneal branches. The nerve sample was preserved in Karnovsky fixative for $24 \mathrm{~h}$, then post fixed in $1 \%$ osmium tetroxide during $4 \mathrm{~h}$ and embedded in epon Ladd LX-112 (Ladd Research Industries Inc., Burlington, Vermont). Semi-thin nerve cross sections $(1 \mu \mathrm{m})$ were cut on a Reichter Ultracut microtome (Reichter, Wien, Austria) and stained with toluidine blue.

\subsection{Image acquisition}

Photomicrographs were taken using a color filter system in a Zeiss microscope with an $40 \times$ PLANAPO oil immersion objective at a magnification of $\times 1650$. A total of 95 photomicrographs (referred to here below as images) were taken, representing a $209 \times 140$ $\mu \mathrm{m}^{2}$ tissue area. A slight overlap between adjacent microscopic frames was adjusted manually to take care of partial fibers lying on the edge of each photomicrograph. Images were then digitized by a Nikon 25-1000 software system with an $1850 \times 1234$ pixel resolution. A microscale $(0.01 \mathrm{~mm}$, Wild, Switzerland) was processed in the same way so as to obtain the scaling factor. The pixel size corresponded to $0.11 \mu \mathrm{m}$. 

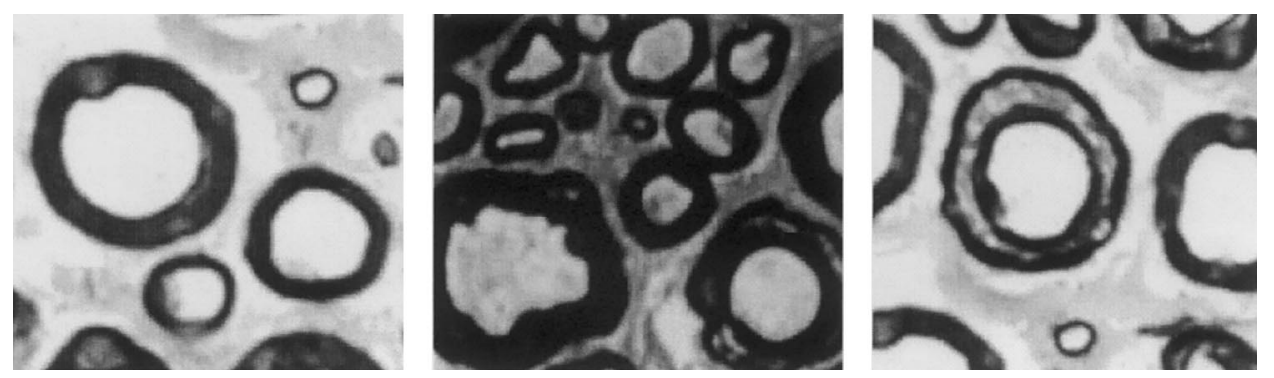

Fig. 1. Typical image illustrating fiber irregularities. Left, illustration of fiber diameter variability (subset size of $22.5 \times 20.5 \mu \mathrm{m}$ ). Center, connection between densely packed fibers (subset size of $24.7 \times 21.8 \mu \mathrm{m}$ ). Right, bright rings in the myelin sheath due to clefts of Schmidt-Lantermann (image subset size of $25 \times 23.1 \mu \mathrm{m}$ ).

\subsection{Basic morphological principles}

A normal mixed nerve such as the sciatic consists of different kind of fibers with different shapes, diameters and distributions. It contains also structures such as capillaries and connective tissue including endoneurial fibroblasts. In this paper, any structure different from nerve fibers will be considered as an artifact. In a correctly preserved, fixed and stained nerve section, the myelin appears as a dark annulus (see Fig. 1). Nerve fibers can be considered as objects with a clear region surrounded by a dark annulus of approximately constant thickness. These objects exhibit a rather round or elliptical shape, but important distortions are frequently observed. Therefore, global recognition criteria such as circularity are not robust. Some decision rules can nevertheless be used on these objects in order to improve object segmentation. A useful parameter is the ratio $P^{2} / A$, where $P$ is the object perimeter and $A$ its area. This can be computed for the axon and the fiber (i.e. the axon surrounded by its myelin sheath). Another useful criterion is the ratio $d / D$, where $D$ and $d$ are the fiber and axon diameter, respectively. It must also be stressed that some deformations may result from the tissue processing technique itself such as the selected fixative or the buffer molarity (Robertson, 1958; Schultz and Karlsson, 1965) or coloration artifacts (see Fig. 1). For this reason, the $P^{2} / A$ ratios have to be adjusted accordingly.

The $d / D$ ratio varies between 0.36 and 0.95 according to Suderland (1968), but for this work only 0.6 value was considered according to Rushton (1951). Unfortunately, fibers also present a number of highly variable features that may hinder the efficiency of detection algorithms. For instance, in a mixed nerve such as the sciatic, fiber diameter varies between about 1 and $30 \mu \mathrm{m}$ (Suderland, 1968). Also, fibers can be either neatly separated or densely packed together. For all these reasons, no additional criterion can be defined regarding fiber size or distribution topology.

\subsection{Image processing}

The image processing is divided in five steps. First, pixels are classified as myelin (black) or non-myelin (white) pixels according to their gray level values. Next, the resulting binary image is simplified using connected morphological operators according to rules derived from the basic morphological principles described above. Third, adjacent fibers are separated using a distance criterion. Fourth, additional morphological criteria are used to detect and discard false fibers. Finally, oblique slices are detected and a geometrical correction is performed, if needed.

\subsubsection{Pixel classification}

Pixel classification aims at classifying the pixels as belonging to a myelin sheath (black) or not (white). Unfortunately, histological sections contain tissue structures with inhomogeneous staining densities. This leads to smooth variations of the average luminance in some regions of the image. Therefore, for every pixel in the image, the threshold level should be computed locally, i.e. based on the analysis of the histogram of a small image subset around it. The size of this subset is set slightly larger than the maximum feature size one expects to find in the image, typically $15 \times 15 \mu \mathrm{m}$. In nerve sections, myelin is darker than the endoneurium and the axoplasm. Thus, a typical image subset histogram has two peaks, sometimes three when the gray level of endoneurium and axoplasm differ.

The threshold level is set to correspond to the minimum between the first two peaks of the smoothed histogram (i.e. the Gaussian filtered histogram with a 65 kernel size). The gray levels for which $15 \%\left(L_{15}\right)$ and $50 \%$ $\left(L_{50}\right)$ of the histogram integral is darker are first computed. $L_{15}$ is a typical value of gray levels within the 'myelin peak'. $L_{50}$ is typical of the 'non-myelin peak'. The average between $L_{15}$ and $L_{50}$ is used as an initial estimation for a local minimum search, which is con- 

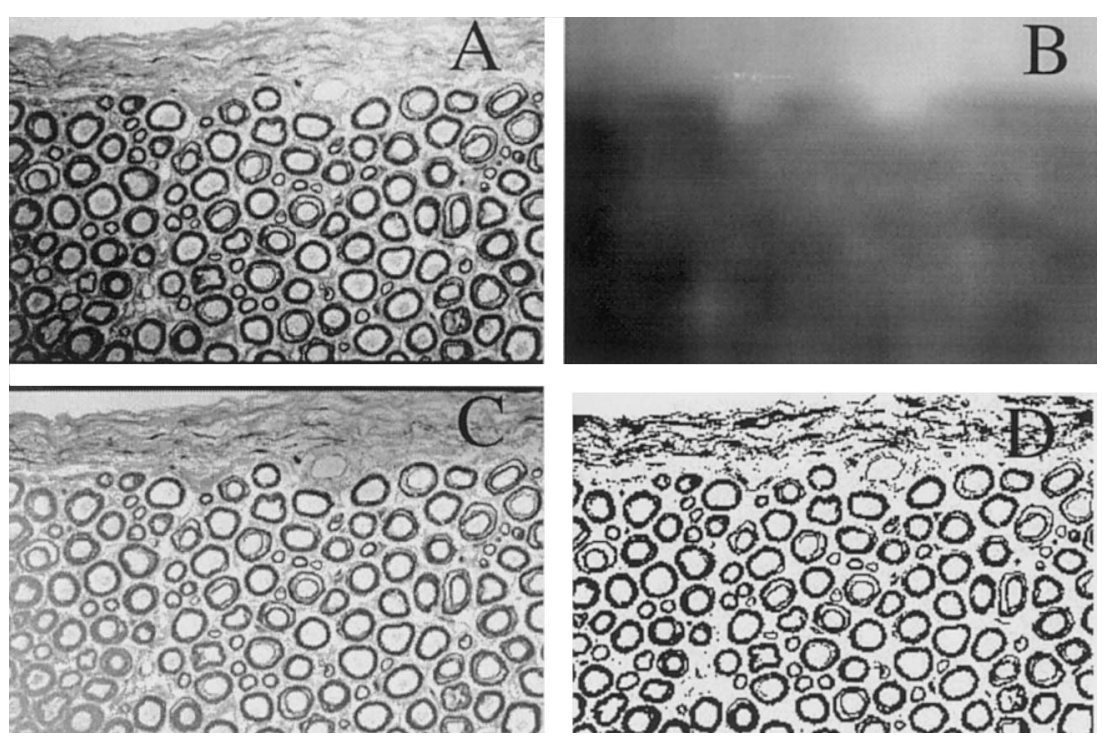

Fig. 2. (A) Original gray level image. (B) Threshold levels, resulting from the analysis of local histograms. (C) Undistorted image, i.e. difference between the original image and the threshold image $(C=A-B+128)$. (D) Binary image resulting from the threshold $(D=(A>B))$.

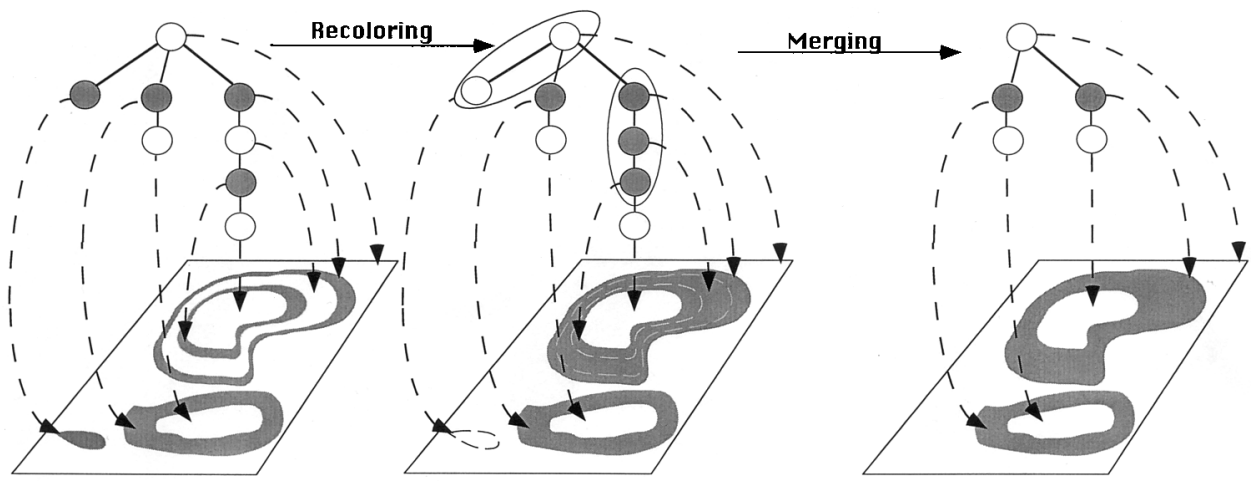

Fig. 3. On top of the three figures are illustrated the zonal graphs (solid lines) associated to each binary image $P(X)$ (dashed lines represent each vertex association to any particular feature in the image). Small spot is a useless feature and thus is removed with the area operator, which flips the binary value of zones with an area of less than $0.5 \mu \mathrm{m}^{2}$. In the zonal graph, its representation corresponds to a black leave, which is re-colored in white and merged with the root vertex (background). For the larger feature, the annulus within the myelin produced by a cleft of Schmidt-Lantermann, corresponds in the zonal graph to a white region surrounded by two black regions and thus the area is re-colored in black and the three vertices are merged together. The third features stands for an actual axon and is left unchanged (adapted from Heijmans, 1999).

ducted on the smoothed histogram ${ }^{1}$. Pixel classification typically resulting from this thresholding procedure are shown in Fig. 2.

In practice, a complete local analysis histogram can not be performed for every image pixel, provided that it is necessary to maintain a low computational cost. Instead, it is only performed on pixels belonging to a square lattice in which the points are $10 \mu \mathrm{m}$ apart. For points not belonging to the lattice, the threshold level is found by bilinear interpolation of the threshold values at the four nearest lattice points.

\footnotetext{
${ }^{1}$ If necessary, the $L_{15}$ and $L_{50}$ values can be adjusted by the experimenter on a single image and extended to all the images of the studied nerve section.
}

\subsubsection{Connected operators filtering}

After the pixel classification step the resulting binary image still contains artifacts, which are easily expressed and handled in terms of regions and their properties. A partition is a subdivision of the image into disjoint zones. The binary image is considered as a partition $P$ $(X)$ of $X$, the set of pixels, into black and white zones.

The zonal graph (examples of which are illustrated on top of the three representations of Fig. 3) is composed of arcs (solid straight lines) connecting vertices (Small black and white disks). Each vertex is associated to disjoint zones of the binary image $P(X)$. A black (white) zone is represented by a black (white) vertex. The zonal graph in binary images is always a tree (no cycles are present). Terminal vertices of a tree are called 
leaves i.e. the regions with only one neighbor. Given two partitions $P$ and $P^{\prime}$ of the image, we say that $P$ is coarser than $P^{\prime}$ if $P^{\prime} \subseteq P$. A morphological operator $\psi$ is called connected if the resulting partition $P(\psi(X))$ is coarser than $P(X)$, for any set $X$, or in other words, connected zones are either left untouched or changed altogether. In the common case where connectivity is based on adjacency, connected operators can be described and implemented by re-coloring and merging vertices of the corresponding zonal graph (Fig. 3). The formalism used here, known as connected morphological operators (Heijmans, 1999), operates on the zonal graph (see Fig. 3 ) of the image rather than at the pixel level. Connected operators only allow certain zones in the image to change color, but zones can not be shifted or broken and no new boundaries can appear, assuring thereby that artifacts disappear without introducing new problems.

The best known connected morphological operator is the morphological opening by reconstruction (Serra, 1982), where objects which are too small to contain the structural element of the original erosion are deleted while large objects are left unchanged. More complex criteria can of course be defined, either considering each zone separately (it is then called a grain operator) or considering the relationships between zones and their neighbors. We will use both hereafter.

Different connectivities yield different zonal graphs. In our case, we used 8-adjancency (a pixel is compared with its eight direct neighbors) for foreground pixels and 4-adjacency for background pixels. This defines a topology similar to the continuous case, and ensures that the zonal graph is actually a zonal tree. A number of connected operators are then applied successively in order to remove the artifacts described in Section 2.3 and to identify fiber candidates.

- Noise in the original image may lead to small mislabeled areas in the binary image. Those are removed by applying the area operator (Fig. 3), that switches the color of all zones whose areas are smaller than a given value. Unfortunately, the area operator is not stable. Applied iteratively it can fail to converge and then oscillates between two solutions. Therefore, we restrict its action to the leaves of the zonal graph. We apply this 'leave area operator' until we reach idempotence $\left(\psi^{2}=\psi\right)$. The surface value for which areas are re-colored is chosen smaller than the smallest axons, for instance $0.5 \mu \mathrm{m}^{2}$ or 40 pixels.

- Fibers always have a bright center surrounded by a black ring i.e. a black region with two neighboring white ones. Therefore, black leaves in the zonal graph do not represent a useful feature and are removed. The 'black leave operator' is applied once.

- Clefts of Schmidt-Lantermann can split the myelin sheath in two parts, as illustrated in Fig. 1 (right panel). In the binary image, this appears as a white ring surrounded by two black rings. White rings are detected by computing the gravity center of all white areas. If the center of a ring is located outside the area itself, two cases can appear, either the ring is open and corresponds to a leaf of the zonal graph or the ring is closed and has two neighbors in the zonal graph. In the former case, the ring is merged with its only neighbor; in the latter, the three vertices of the graph are merged together as a black area, i.e. the ring is re-colored in black.

Finally, according to the range of fiber diameters (Suderland, 1968) and the ratio $d / D$ of 0.6 (Rushton, 1951), axon candidates are identified as white leaves in the zonal graph satisfying a size criterion $(1.2<d<18$ $\mu \mathrm{m})$. Moreover, to guarantee the compactness and approximate circularity of the center of the axon, the ratio $P^{2} / A$ is kept below a certain level to be adjusted by the experimenter (typically 12).

\subsubsection{Separation of connected fibers}

Unless fibers are very sparse in the binary image, some of them will appear connected in the resulting image. In the zonal graph, this means that several white leaves that are axon candidates share the same black vertex neighbor. This section deals with the division of the corresponding black zone into subregions that are either myelin sheaths surrounding axon candidates or artifacts to be merged with the background.

Consider first a single white area. The thickness of the myelin sheath around it is evaluated as follows, $X_{d}$ is defined as the set of pixels at a distance $d$ of a set $X$ of pixels

$X_{d}=\left(X \oplus S_{d+1}\right) \cap\left(X \oplus S_{d}\right)^{c}$

with $S_{d}$ being a circular structural element of size $d$ and $\oplus$ being the Minkowski addition. The typical thickness of the myelin sheath around a white area $X$ is then defined as the smallest distance $d$ for which there are more white than black pixels in $X_{d}$. This can be very efficiently implemented using the approximate Euclidean distance transformation (EDT) by propagation (Ragnelman, 1992) or the exact EDT (Cuisenaire, 1999). These algorithms compute distance maps, i.e. images where the value of each pixel is the Euclidean distance from this pixel to a set of pixels $X$, that is the shortest distance from that pixel to a pixel of the set $X$, initially, the map is set to 0 for pixels of $X$ and to the maximum integer for all others, and pixels of $X$ are stored in a dynamic list. Then, for each pixel of the list, the nearest pixel of $X$ is considered as the nearest pixel of $X$ for its neighbors. If this leads to a smaller distance value than that currently in the map, this value is updated and the neighbor is inserted in the list. In order to reduce computational cost, pixels in the list must be treated by increasing distance value order rather than in a simple FIFO order. This is done by replacing the single list by a number of buckets corresponding to each possible 


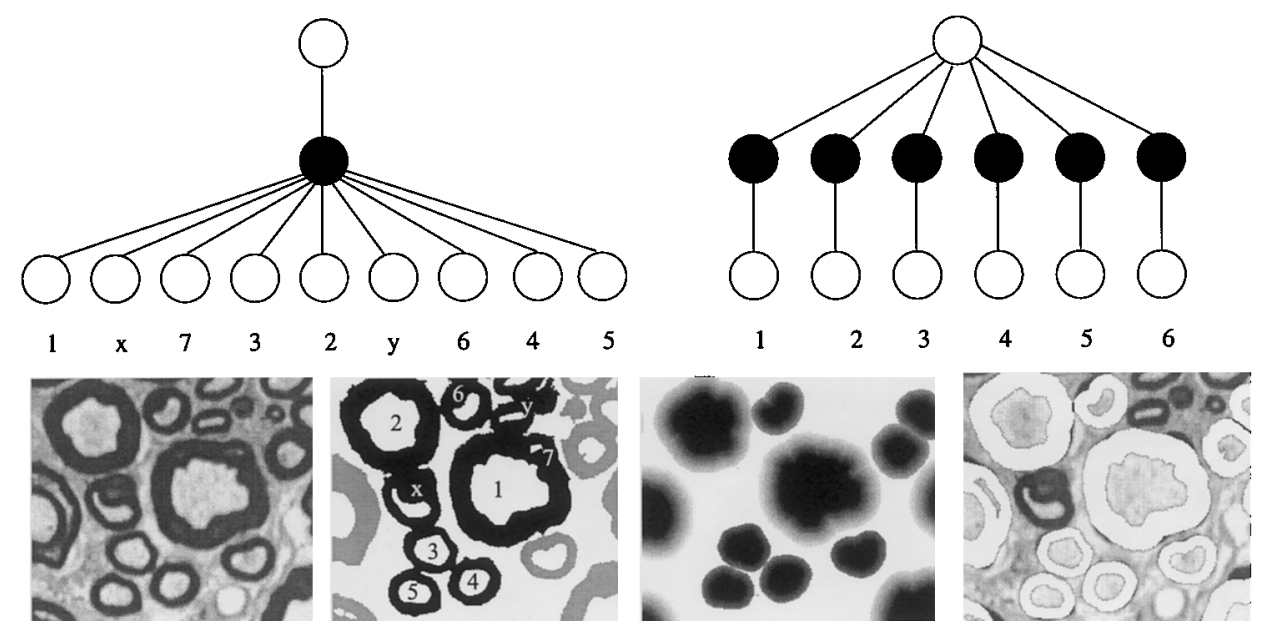

Fig. 4. Upper figure, zonal graphs of the region of interest before (left) and after (right) axon separation. Lower figure, axon separation by distance transform (image subset size of $31.5 \times 27.3 \mu \mathrm{m}$ ). From left to right, original image; result of the connected operators filtering (the black area is a connected area that needs to be split into several fibers); distance map, fiber candidates are examined by size order from larger fibers to smaller ones (Euclidean distance transformation is propagated from the axon toward the periphery up to the white/black pixel ratio is larger than 0.8); resulting fibers (represented as white annuli) overlaid on the original image.

distance value, or even better, by the square of each possible value, in order to consider only integers. Buckets are then emptied by increasing distance value order. With this algorithm, the set $X_{d}$ of pixels at a distance $d$ of $X$ is the set of all pixels present in the buckets after bucket $d$ has been processed. The number of black and white pixels in $X_{d}$ is dynamically computed for every distance $d$ and the propagation process stopped as soon as the termination condition is reached. The pixels that were reached by the propagation process - and only those - are considered as belonging to the myelin sheath around the axon candidate.

Consider now all the 'less axon candidate' areas, which are leaves of the same black area in the zonal graph. The previous procedure is applied to each candidate, in decreasing size order. An illustrative example is given in Fig. 4. Among the nine leaves of the graph, leaves $x$ and $y$ have been discarded at the previous stage, because they lack circularity to be proper fiber candidates. Among the seven remaining candidates, areas 1 and 6 are true fibers while area 7 is a cleft of Schmidt-Lantermann. In the black area itself, some pixels correspond indeed to myelin while others result from an artifact. The algorithm efficiently separates fibers numbered from 1 to 5 . For area number 6 , the propagation process reaches pixels that were previously considered as belonging to the myelin sheath around area 2. These pixels are relabeled as belonging to the sheath around the axon they are closest to. The resulting arc between the two fibers corresponds either to the thickness of the smallest fiber, or to the iso-distance between the two white areas. Area number 7 is included inside the previously computed myelin sheath around area 1 and therefore it is discarded.

\subsubsection{False positive detection}

The above procedure to detect fibers can lead to two types of error, missed detection when a true fiber is not found and false positive when an image feature is wrongly considered to be a fiber. False positives are considered a worse problem since they are most likely to introduce a bias in the fiber distribution statistics, as most false positives are small in size. Missed detection is only detrimental if its rate is size dependent, and if their number reaches a significant percentage of the total.

In order to minimize the number of false positives, two measures are considered. These are an intrinsic reliability measure for each fiber and a conflict measure that uses information from the surrounding features. The reliability measure depends mostly on a $d / D$ ratio reasonably close to 0.6 (Rushton, 1951). The combination of fascicle edge and isolation criteria gives the conflict measure. For densely packed fibers, false positives often occur in the space between three neighboring axons. These are detected by looking at the pixels on their edges. Most of those pixels are considered as belonging to other axons, while they really belong to the background. On the other hand, in images including the edge of the fascicle, some external features can be wrongly taken for fibers. Those are detected since they are isolated from the rest of the detected fibers.

\subsubsection{Correction of obliquity}

Even in expert hands a perfect transversal cut is almost impossible, and a certain degree of obliquity always remains. In that case, most fibers appear as ovals instead of disks. Oblique transversal sections are detected by inspecting the principal axis of fibers, when 
most of the fiber long axes are aligned (see Fig. 5). Although, in these cases the myelin sheath is thicker at the ends of the longer axis than at the shorter one, this parameter is highly irregular and not stable enough to be useful for obliquity correction. Fiber orientation provides a better estimation of the section's obliquity. A general index of the image obliquity is obtained by calculating the obliquity vector $\vec{v}_{i}$ for the $i$ th fiber, which is defined as follows:

$\vec{v}_{i}=\left|v_{i}\right| \mathrm{e}^{j \theta_{i}}$

where $\theta_{i}$ is the angle of the longest axis with the horizontal and $\left|v_{i}\right|$ is

$\left|v_{i}\right|=\frac{\text { longest fiber axis }- \text { shortest fiber axis }}{\text { shortest fiber axis }}$

A general measure over a number $N$ of fibers is

$v_{\text {mean }}=\left|v_{\text {mean }}\right| \mathrm{e}^{j \theta_{\text {mean }}}$ where

$\left|v_{\text {mean }}\right| \mathrm{e}^{j 2 \theta_{\text {mean }}}=\sum_{i=1}^{N} \omega_{i}\left|v_{i}\right| \mathrm{e}^{j 2 \theta_{i}}$

and

$\left|v_{i}\right| \mathrm{e}^{j 2 \theta_{i}}=\frac{\vec{v}_{i}^{2}}{\left|v_{i}\right|}$

with weighting factors $\omega_{i}$. In practice, we use $\omega_{i}$ as the shortest fiber axis length. Thus, to correct the obliquity of the cut, all fibers are contracted along the direction $\theta_{\text {mean }}$, the mean long axis by a factor $1 /\left(1+\left|v_{\text {mean }}\right|\right)$.

\section{Results}

The assessment of the present method was structured in four different ways.
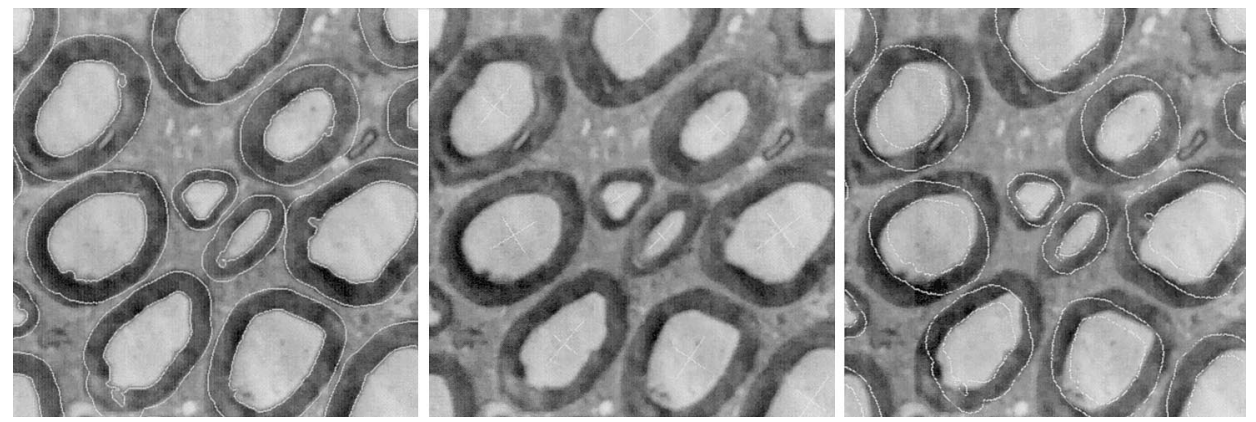

Fig. 5. Correction of obliquity (image subset of $50 \times 51 \mu \mathrm{m}$ ). From left to right, fibers found on an oblique section, principal axes of fibers and oblique-corrected fibers.
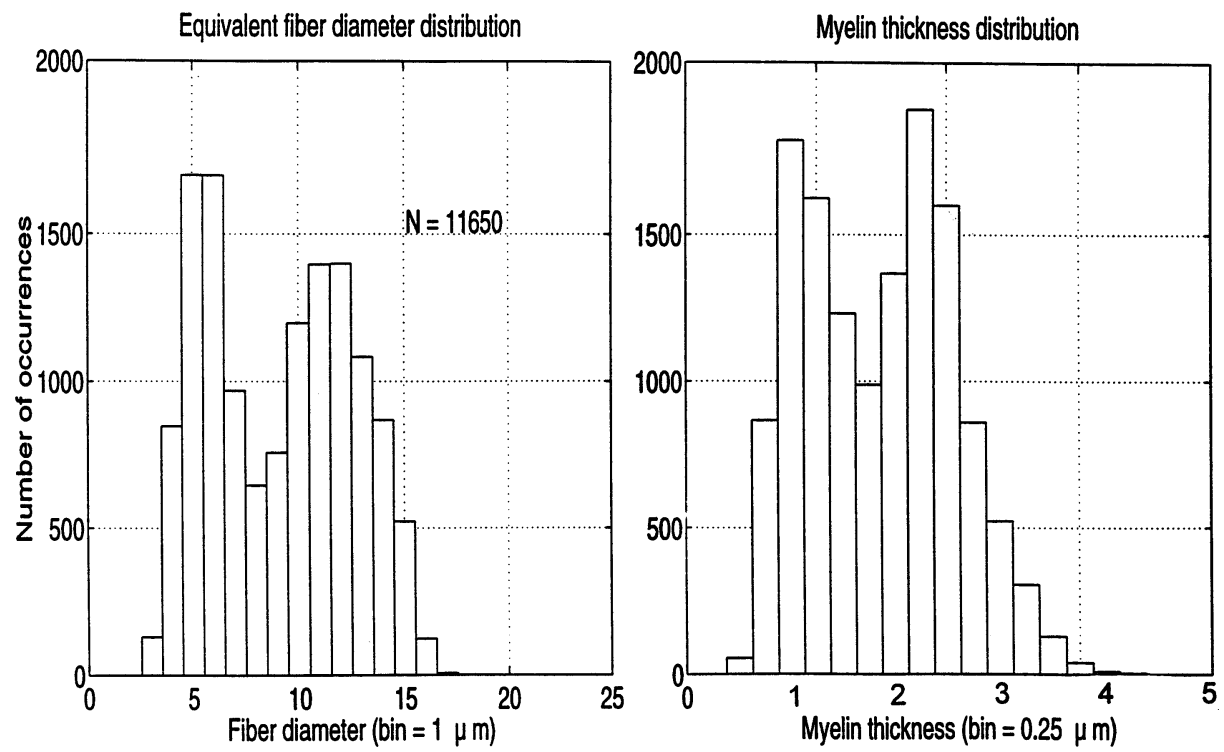

Fig. 6. Illustration of fiber diameter and myelin thickness histograms constructed from available automatic data. 


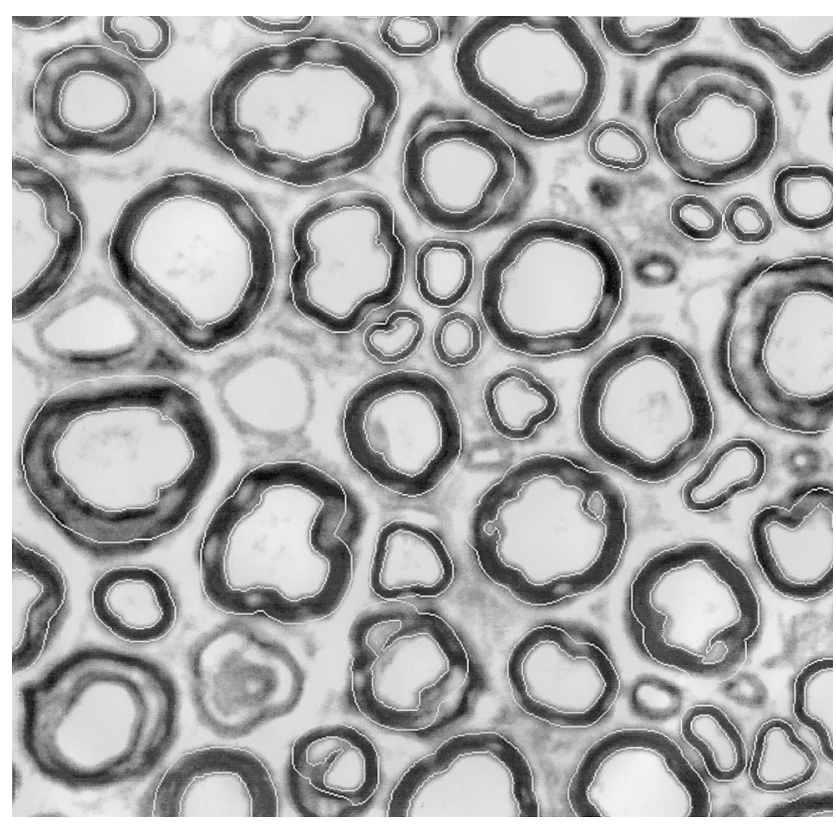

Fig. 7. Detected fibers overlaid upon part of a typical image (image subset of $68 \times 88 \mu \mathrm{m})$.

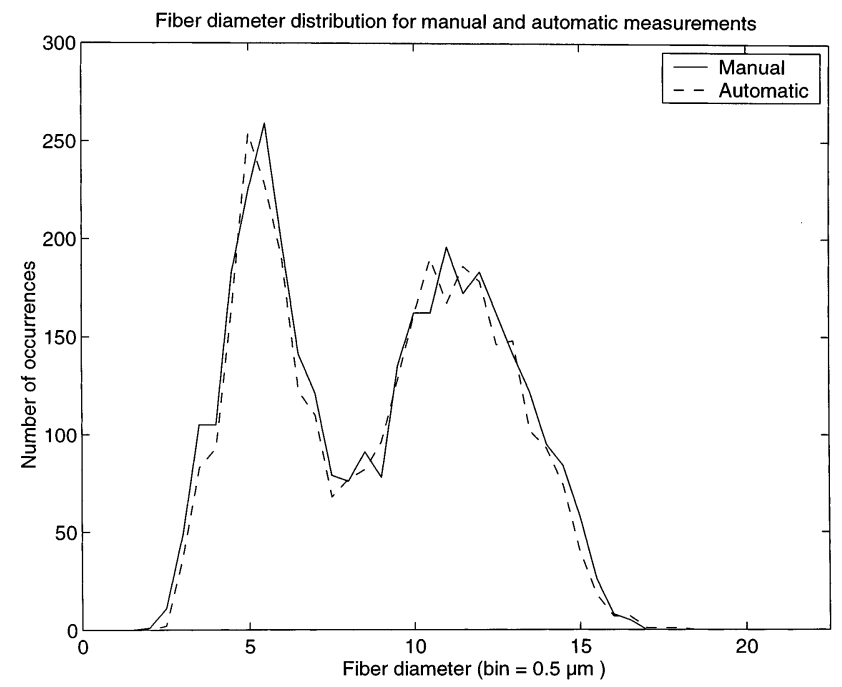

Fig. 8. Distribution comparison of automatic and manual measures for equivalent fiber diameters (bin width, $0.5 \mu \mathrm{m}$ ).

\subsection{Missed detection and false positives}

Firstly, for the lattice configuration described in Section 2.4.1, the total number of recognized fibers, missed detection and false positives were evaluated in the whole image set. The total number of fibers was found to be 11650 . Fiber diameter and myelin thickness histograms were constructed from these data and are illustrated in Fig. 6.

The detected fibers can be visually evaluated by superimposing on the original image the algorithm estimation (Fig. 7). From the total image set, 783 fibers were found to be missed detections, and from a total of
11650 fibers, 113 were considered as false positives. Of the 783 missed detections, 427 corresponded to fibers with a diameter below $7 \mu \mathrm{m}$. Hence, estimating the real number of fibers as $12320(=11650+783-113)$, over the entire available surface, $0.92 \%$ of false positives and $6.36 \%$ of missed detections appeared. From these missed detection $47.2 \%$ belonged to the smaller group diameter. By comparison, among the 11650 fibers automatically detected, $39.7 \%$ (4628 fibers) had a diameter below $7 \mu \mathrm{m}$. From the 427 small profiles, 273 (64\%) were detected in the first classification state, but were ruled out by the geometrical relationships defined in Section 2.3 and from the 356 large profiles, $245(69 \%)$ were also excluded in this state.

\subsection{Comparison with manual measurements}

Here, the accuracy of the measurements themselves was evaluated. Twenty images (about $18 \%$ of the available nerve surface) from the same nerve section were selected by lottery, but no image was selected more than once (i.e. sampling without replacement). Using a standard software (NIH image for MACINTOSH), contour fibers were manually drawn and measured. A total number of 3434 fibers were found manually compared to 3256 obtained by the automatic method, from which 33 were considered as false positives and 211 as missed detections. This amounts to a $6.14 \%$ error recognition rate only on these selected images, which is typical of the error rate found for the whole data set.

In Fig. 8 the corresponding manual and automatic histograms are depicted. This figure shows a good fit for both peaks. Histograms do not differ significantly $\left(\chi^{2}=18.89\right)$. Here, the null hypothesis is that both data sets are drawn form the same population distribution function at a $\alpha$-significance level of $5 \%$, and the $P$-value is interpreted as answering this question, if the null hypothesis is true, what is the chance of randomly obtaining a larger discrepancy between both distributions?

This slight difference between automatic and manual measures can be better visualized in the myelin thickness distribution, drawn as relative cumulative frequency histograms. As illustrated in Fig. 9, the pattern of both curves is similar with a slight right shift of the manual curve for thickness larger than $2.5 \mu \mathrm{m}$.

\subsection{Signal-noise ratio analysis}

Third, the noise introduced by the automatic procedure is compared to the variability introduced by noise sampling. The evaluation is always performed on the 20 image group. We proceed as follows, the set of 20 images is randomly split into an arbitrary number of pairs of two subgroups of ten images, which in turn is compared for manual and automatic measures. Fig. 10 


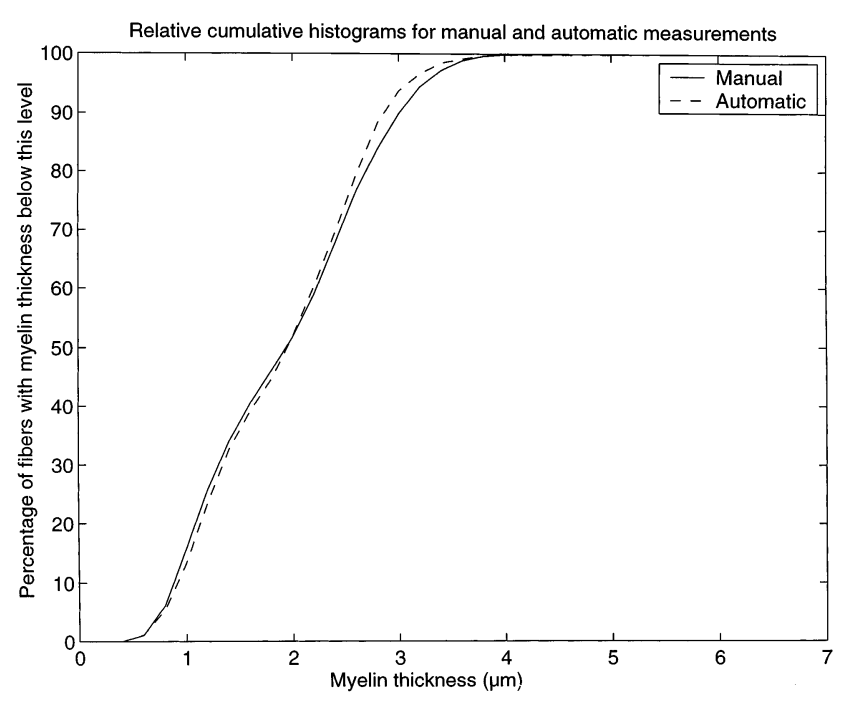

Fig. 9. Relative cumulative frequency histograms for myelin thickness.

shows the fiber distributions found for two particular subgroups with manual and automatic procedures.

The $\chi^{2}$-test on these histograms reflect differences in the data distributions. The procedure is repeated here 1000 times, splitting the data set into two arrays of ten images each time. The $\chi^{2}$-test is calculated for each
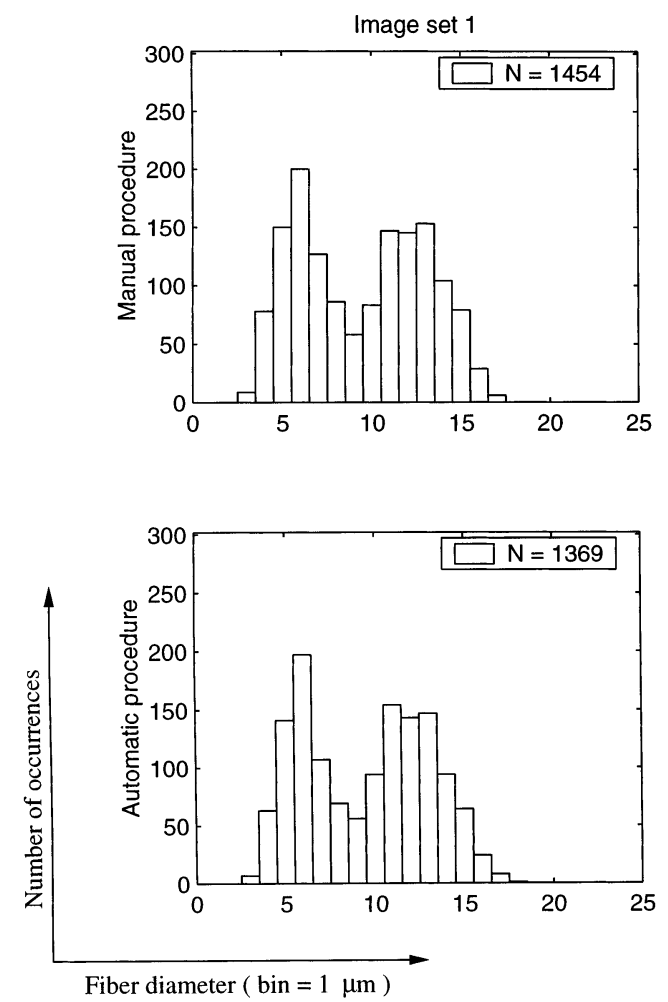

distribution data pair (four in total). Three data groups are plotted in Fig. 11 (logarithm scale). On the left graph $\chi^{2}$ values between manual measures for subgroups 1 and 2 appears drawn against $\chi^{2}$ values between manual and automatic measures of subgroups 1 and 2. As it can be observed in the left graph, there exists a clear trend of the manual-manual differences (differences due to sampling) to be larger than the manual-automatic differences. One might objects that the lower $\chi^{2}$ values result from a smoothing effect of the automatic procedure on the histograms. The right graph answers this objection, we observe that automatic-automatic differences are as large as manualmanual differences.

\subsection{Obliquity correction}

Finally, the obliquity correction was carried out in some images. Typical results are shown in Fig. 5. The principal axis (central image), indicates the orientation of each fiber. The correction is shown in the right image. In this case, the computed mean long axis appears to be $36 \%$ longer than the small axis, which corresponds to a $42^{\circ}$ obliquity. On the other hand, for cuts that obviously appear to be nearly perfect, the suggested correction ratio is usually close to $0.8 \%$, i.e. negligible.
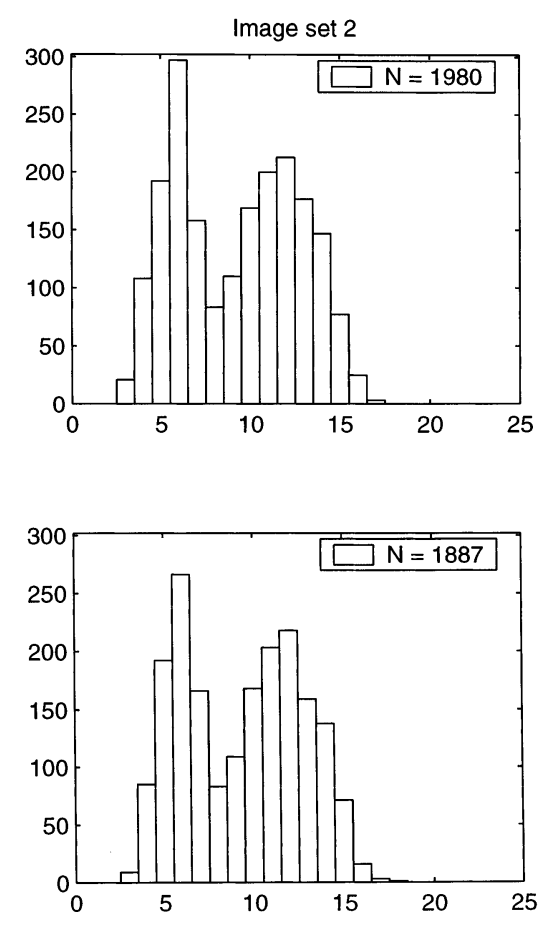

Fig. 10. Comparison of fiber distributions for a random arrangement of the 20 images into two data sets, 1 (left) and 2 (right) for manual (above) and automatic (below) measurements. Histogram differences between the manual subsets 1 and 2 were found to be larger $\left(\chi_{M_{1}}^{2}-M_{2}=114.04\right)$ than histogram differences between the manual and automatic for the first data set $\left(\chi_{M_{1}-A_{1}}^{2}=10.57\right)$ and than for the second data set $\left(\chi_{M}^{2}-A_{2}=\right.$ 14.00). Automatic differences for data sets 1 and 2 were also important $\left(\chi_{A_{1}-A_{2}}^{2}=108.93\right)$. 

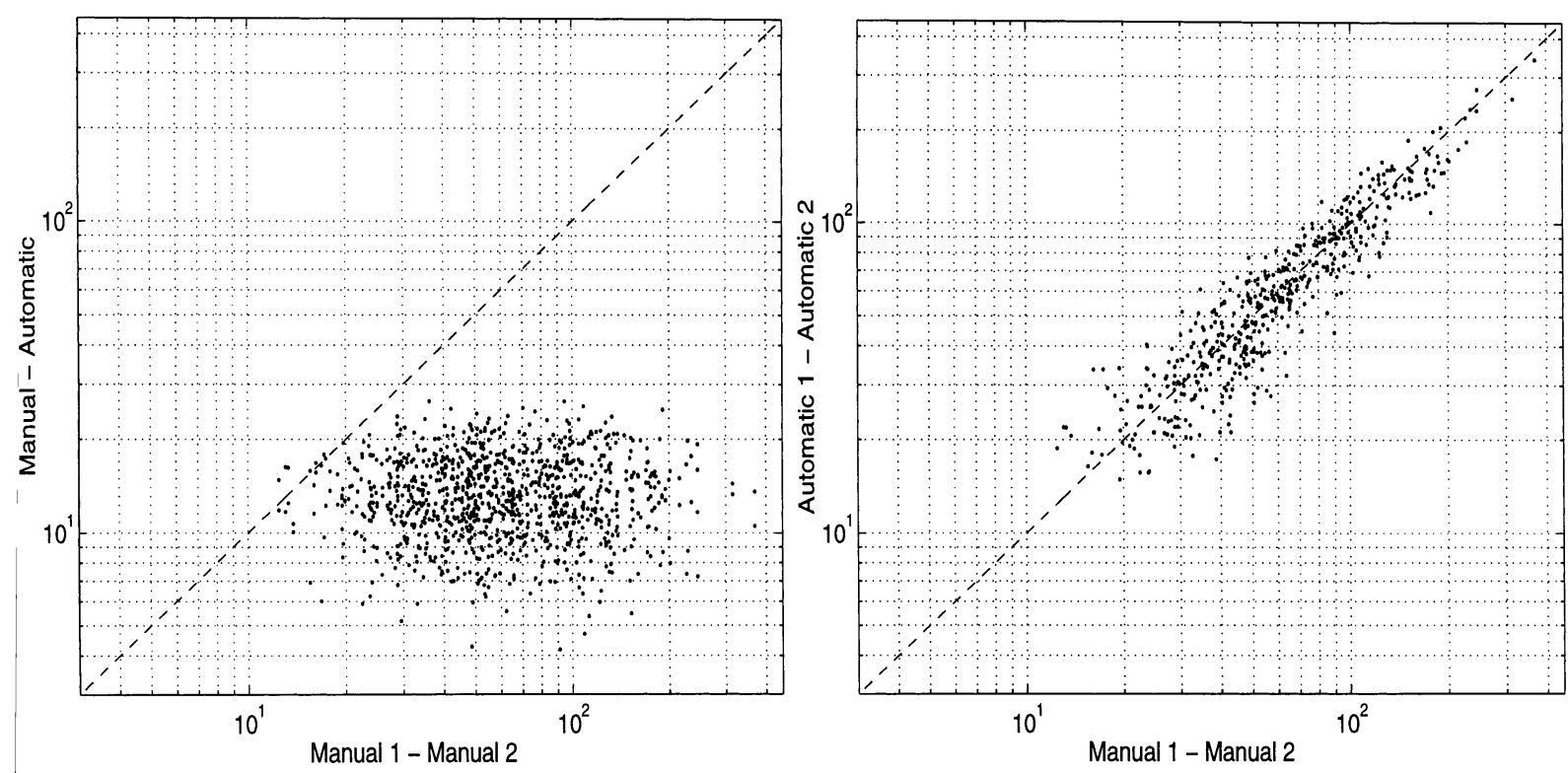

Fig. 11. Sampling-measurement accuracy noise ratio. The data set of 20 images is shuffled and divided into two subsets of ten images. Histogram distributions for manual and automatic data are computed for each subset and $\chi^{2}$ values calculated for each different pair distribution (manual and automatic). The left graph shows $\chi^{2}$ values for manual-manual distributions depicted against $\chi^{2}$ values for manual-automatic distributions. In right graph manual-manual $\chi^{2}$ histogram values are drawn against automatic-automatic $\chi^{2}$ histogram values.

\section{Discussion}

This study describes an efficient automatic method to measure various fiber parameters such as the total number of fibers, fiber diameter, fiber perimeter and myelin thickness distributions within a nerve trunk. As it was pointed out before, morphometrical analysis has evolved from purely manual methods in which a complete tracing was needed with all side errors introduced by the experimenter and by the few profiles able to examine, to more intelligent sampling schemes which have reduced considerably the manual work. In this context an automatic method is of course the best choice to maximize efficiency and minimize work time, provided its accuracy is proven. The method presented here is fully automatic once the various parameters have been set to appropriate values. These values are constant for a given fixation, coloration and acquisition procedure. In practice, the experimenter selects the appropriate settings for a single image and applies them to the full image set of the same nerve. One advantage with this automatic image analysis approach is that no particular definitions of the staining intensity of the fiber are included, but it is the local histogram properties that defines them. In addition, this threshold election permits to conserve the original axon shape and to recognize any fiber contour. Also, this method incorporates classical rules such as diameter ratio between the axon and the fiber, or axon perimeter bounds, and allows them to be expressed as simple morpho-mathematical criteria.
Although, some fiber axons were missed and a few artifacts were taken as actual fibers, comparison of manual and automatic methods in about $18 \%$ of the available nerve surface, with a standard $\chi^{2}$-test did not show any significant difference. In this sample, slight difference were found in large fiber measures. This can be attributed to the fact that a manual operator follows intuitively the exact fiber boundary while the program follows a constant myelin thickness hypothesis (which one of these two hypothesis is the most correct is open to discussion). Besides, it must be stressed that manual measures are subjected to many error sources such as subjective decisions, time of measure, or inter-observer variability, etc. In other words, manual measures always keep an error margin that can contribute to these slight differences.

A very important feature of the method is the choice of the local threshold levels. For instance, a previous version of the software in which the image subset was set to $25 \times 25 \mu \mathrm{m}$ size led to a higher missed detection ratio for small fibers $(4.09 \%$ instead of $3.47 \%$ of the present version) because the local contrast was badly evaluated. In that case, small profiles had a higher probability to be missed. In general, there are two possible error sources, the first is an inappropriate choice of the local threshold and the second is the fiber deformation which makes them different from our model of fiber. Indeed, the small fibers tend to be missed because their shape variability is higher than that of larger ones. Unfortunately, relaxing shape criteria may 
not be an appropriate response to this problem since it might introduce new artifacts.

Whether or not a given method is useful in practice depends on its 'noise' in comparison to the 'signal'. A well-designed experiment is one in which the signal is greater than the noise. In our case, the noise is the difference between manual and automatic measurements for the same images. In most biomedical papers, the relevant signal is the intrinsic variation between animals. Mayhew and Sharma (1984a) has demonstrated that the noise introduced by sampling was reasonably low for practical purposes. In this paper, we show that the noise introduced by the automatic method is well below the variability introduced by a random sampling scheme. By transitivity, the noise introduced by our method is likely insignificant compared to the relevant signal.

As it can be observed in Fig. 11, $\chi^{2}$ values for the manual distributions in the two random subgroups systematically lead to larger values than the comparing $\chi^{2}$ values for the manual-automatic distributions. In the experiment of Section 3.3 each subgroup ranged from $7.3 \%$ (all boundary images are in the same subgroup) to $9.6 \%$ of the available nerve section, which is usually considered (Mayhew and Sharma, 1984a) an adequate representation of the total population. In these conditions, it is valid to deduce that the noise introduced by sampling is more important than the one introduced by the method measurement. A fortiori, the noise introduced by the automatic method is clearly within reasonable bounds for practical studies.

A mixed nerve such as the sciatic is the proper specimen to evaluate the method due to the wide variability in fiber sizes, shapes and distribution. Besides, the number of problems found on these images are common to any peripheral nerve, provided that good fixation and staining protocols allow to obtain images with adequate contrast. Of course, the staining intensity and contrast can vary from nerve to nerve or even within different regions of the same nerve section, but our method shows sufficient robustness to these luminance changes. Thus, a practical conclusion from this study is that despite the available limited data, the accuracy of the method is acceptable for morphometric studies.

Although, the missed detection rate represents about $6 \%$ of the number of fibers, this problem seems to be a minor one since in our small ten image subgroups, we did not find significant differences between manual-automatic measures. Indeed, if necessary, the missed detection ratio could be lowered by interactively pointing to the missed detected fibers and forcing the corresponding leaves in the zonal graph to become fiber candidates and to be considered first in the fiber separation process. For the 95 images of the present study, the missed detection was $6.36 \%$, which does not seem to represent hard work for an experimenter. False positives, which originate from some defects in endoneurium coloration or from light spots inside myelin sheaths, were also present at a negligible level $(0.92 \%)$. Obviously, if necessary, the false positive rate can be brought down to zero with an operator pointing at those errors.

Obliquity introduces a bias in morphometrical measures. For instance, a tilt of $25^{\circ}$ overestimates the areas by $10 \%$. The applied obliquity correction is an effective means to overcome this bias. It equals the two principal axes, taking the shortest one as the real diameter of the fiber. Implicitly, we assume that all fibers should be perfect disks. Practically, they display a variety of shapes in a histological stained section, but most of them are almost round. As the correction is averaged over the entire section, the bias induced by a few non-round fibers may be considered negligible. This type of operator interaction is much inferior to that of usual manual methods.

The computational cost is a critical parameter, especially if one wants to introduce interactivity in the process. With this method, most of the processing is done on the zonal graph instead of the image. Because the zonal graph is orders of magnitude smaller than the image, the corresponding processing time is negligible. The most costly stages of the process are then thresholding, creation of the zonal graph and separation of the axons by distance transformation. Graph creation requires labeling of the image and linking of neighboring areas, for which a couple of raster scans are needed. The computation of dilations by Euclidean distance transformation requires one or two passes over each pixel in the propagation area. In total, the whole processing has a complexity linearly proportional to the number of pixels in the image. On a Pentium II at $400 \mathrm{MHz}$, the processing time for each $1850 \times 1234$ pixel image was 15 $\mathrm{s}$, which means a complete nerve can be processed in less than half an hour without operator intervention. In contrast, manual measurements for the same image size, took between 55 (165 fibers) and $90 \mathrm{~min}$ (252 fibers).

In conclusion, a method to detect automatically neuronal fibers has been designed. Because those fibers are defined from two objects - a white center surrounded by a black ring - the zonal graph and the connected morphological operators are appropriate tools to isolate good axon candidates. The evaluation of the myelin sheath thickness and the separation of aggregate fibers are performed using distance propagation until a given criterion on the propagation front is reached. The experimental results show a good accuracy of the method, at a reasonable computational cost.

\section{Acknowledgements}

This work has been partially supported by grant \# BMH4-CT-96-0897 of C.U.E. (BIOMED program), grant \# 3.4584 .98 of the Belgian FMSR and the 
Belgian FRIA fund (Fonds pour la Formation à la Recherche dans 1'Industrie et dans 1'Agriculture). The authors wish to thank the two anonymous reviewers for their comments, which have helped us focus the biomedical relevance of this method.

\section{References}

Amini AA, Weymouth TE, Jain RC. Using dynamic programming for solving variational problems in vision. IEEE Trans Pattern Analysis Machine Intelligence 1990;12:855-67.

Buchnal F, Behse F. Sensory action potentials and biopsy of the sural nerve in neuropathy. In: Proceedings of the International Symposium on Peripheral Neuropathies. June 26-28, 1978, Milan.

Cragg GB, Thomas PK. Changes in conduction velocity and fibre size proximal to peripheral nerve lesions. J Physiol London 1961;157:315.

Cuisenaire O. Distance transformations: fast algorithms and applications to medical image processing. Ph.D. thesis, Université catholique de Louvain, B-1348, Louvain-La-Neuve, Belgium, 1999, available at http://www.tele.ucl.ac.be.

Donovan A. The nerve fibre composition of the cat optic nerve. J Anat 1967;101:1-11.

Duncan D. A relation between axon diameter and myelination determined by measurement. J Comp Neurol 1934;60:437-71.

Dunn FR, O'Leary DP, Kumley WE. Quantitative analysis of micrographs by computer graphics. J Microsc 1975;105:205-13.

Dyck PJ, Karnes J, O'Brien P, Nukada H, Lais A. Spatial pattern of nerve fiber abnormality indicative of pathologic mechanism. Am J Pathol 1984;117:225-38.

Elmoataz A, Schüpp S, Cloaurd R, Herlin P, Bloyet D. Using active contours and mathematical morphology tools for quantification of immunohistochemical images. Signal Processing 1998;71:21526.

Fernand VS, Young JZ. The size of the fibres of muscle nerves. Proc R Soc 1951;139:299-304.

Fok YL, Chan JCK, Chin RT. Automated analysis of nerve-cell images using active contour models. IEEE Trans Med Imaging 1996;15:353-68.

Garbay C. Image structure representation and processing: a discussion of some segmentation methods in cytology. IEEE Trans Pattern Analysis Machine Intelligence 1986;8:140-6.

Gundersen HJG. Stereology of arbitrary particles: a review of unbiased number and size estimators and the presentation of some new ones, in memory of William R. Thomsen. J Microsc 1986;143:3-45.

Gutmann E, Sanders FK. Recovery of fiber numbers and diameters in regeneration of peripheral nerves. $\mathbf{J}$ Physiol London 1942;43:101-489.

Heijmans HJAM. Connected morphological operators for binary images. Comput Vision Image Understanding 1999;73(1):99-120.

Jacobs JM, Love S. Qualitative and quantitative morphology of human sural nerve at different ages. Brain 1985;108:897-924.

Jain AK, Smith SP, Backer E. Segmentation of muscle cell pictures: a preliminary study. IEEE Trans Pattern Analysis Machine Intelligence 1980;2:232-42.
Kupfer C, Chumbley L, Downer L. Quantitative histology of optic nerve, optical tract and lateral geniculate nucleus of man. J Anat 1967;101:393-401.

Larsen J. Stereology of nerve cross sections. J Neurosci Methods 1998;85:107-18.

Mathieu O, Cruz-Orive LM, Hoppeler H, Weibel ER. Measuring error and sampling variation in stereology: comparison of the efficiency of various methods for planar image analysis. J Microsc 1981;121:75-88.

Mayhew TM. An efficient sampling scheme for estimating fibre number from nerve cross sections: the fractionator. $J$ Anat 1988;157:127-34.

Mayhew TM, Sharma AK. Sampling schemes for estimating nerve fibre size. I. Methods for nerve trunks of mixed fascicularity. J Anat 1984a;139:45-8.

Mayhew TM, Sharma AK. Sampling schemes for estimating nerve fibre size. II. Methods for unifascicular nerve trunks. J Anat 1984b;139:59-66.

Müller AE, Cruz-Orive LM, Gehr P, Weibel ER. Comparison of two subsampling methods for election microscopy morphometry. J Microsc 1981;1231:35-49.

Ohnishi A, Schilling K, Brimijoin WS, Lambert EH, Fairbanks VF, Dyck P. Lead neuropathy: morphometry, nerve conduction and choline acetyltransferase transport. New finding of endoneurial edema associated with segmental de-myelination. J Neuropathol Exp Neurol 1977;36:499-518.

Pick J. Myelinated fibers in gray rami communicants. Anat Rec 1956;126:395-414.

Ragnelman I. Fast erosion and dilation by contour processing and thresholding of distance maps. Pattern Recognition Lett 1992;13:399-409.

Robertson JD. Structural alterations in nerve fibres produced by hypotonic and hypertonic solutions. J Biophys Biochem Cytol 1958;4:349-64.

Rushton WAH. A theory of the effects of fiber size in medullated nerve. J Physiol 1951;115:101-22.

Schultz RH, Karlsson ULI. Fixation of the central nervous system for electron microscopy by aldehyde perfusion. II. Effect of osmolarity, $\mathrm{pH}$ of perfusate, and fixative concentration. J Ultrastruct Res 1965;12:187-206.

Serra J. Image Analysis and Mathematical Morphology. New York: Academic Press, 1982.

Suderland S. Peripheral nerve fibres. In: Suderland S, editor. Nerves and Nerves Injuries. Livingstone: Eyre \& Spottiswoode, 1968:910.

Thiran JP, Macq B. Morphological feature extraction for the classification of digital images of cancerous tissues. IEEE Trans Biomed Eng 1996;43:1011-9.

Torch S, Stoebner P, Usson Y, D'Aubigni D, Saxod R. There is no simple adequate sampling scheme for estimating the myelinated fibre size distribution in human peripheral nerve: a statistical ultrastructural study. J Neurosci Methods 1989;27:149-64.

Treff WM, Meyer-Köning E, Schlote W. Morphometric analysis of a fibre system in the central nervous system. J Microsc 1972;95:337-43.

Wendell-Smith CP, Williams PL. The uses of teased preparations and frozen sections in quantitative studies of mammalian peripheral nerve. Q J Microsc Sci 1959;101:43-54. 